\title{
RESPONSE OF THREE SUGAR BEET VARIETIES TO COMPOST, MINERAL NITROGEN FERTILIZER AND THEIR COMBINATION UNDER SANDY SOIL CONDITIONS II- YIELD AND QUALITY
}

(Received:8.9.2011)

\author{
By \\ S. A. Safina and E. M. Abdel Fatah* \\ Agronomy Department, Faculty of Agriculture, Cairo University, Egypt and * Sugar Crops Research \\ Institute, Agriculture Research Center, Giza, Egypt
}

\begin{abstract}
Two field experiments were carried out at the Agricultural Experiments Desert Station, Faculty of Agriculture, Cairo University in Wadi El-Natroon, El-Beheira Governorate, during 2008/2009 and 2009/2010 seasons, to study the response of three sugar beet varieties, i.e. KWS1436, Swello and Faraha growing in sandy soil to compost (CM), Mineral-N fertilizer and their combinations, at five treatments : 4 tons fed ${ }^{-1}$ of $(\mathrm{CM}), 4$ tons fed ${ }^{-1}$ of $(\mathrm{CM})+80 \mathrm{~kg} \mathrm{~N}^{-1} \mathrm{fe}^{-1}(100 \% \mathrm{~N}), 4$ tons fed $^{-1}$ of $(\mathrm{CM})+60 \mathrm{~kg} \mathrm{~N} \mathrm{fed}^{-1}$ $(75 \% \mathrm{~N}), 4$ tons fed ${ }^{-1}$ of $(\mathrm{CM})+40 \mathrm{~kg} \mathrm{~N} f e d^{-1}(50 \% \mathrm{~N})$ and $80 \mathrm{~kg} \mathrm{~N}^{-1}(100 \% \mathrm{~N})$ on yield and quality of sugar beet under drip irrigation system. The obtained results revealed that sugar beet varieties differed significantly in all studied traits in both seasons except for, sugar yield, purity $\%$ and sugar losses to molasses (SLM) \% in the $1^{\text {st }}$ season only. KWS1436 variety surpassed the other two varieties in sucrose $\%$, extractable sugar $\%$ as well as sugar yield, also, it gave the highest root yield $\left(28.81\right.$ ton $\left.\mathrm{fed}^{-1}\right)$ and purity in the $1^{\text {st }}$ season. Swello variety recorded the highest root yield (29.96 ton fed $\left.{ }^{-1}\right)$, in the $2^{\text {nd }}$ season. In combination treatments, increasing $\mathrm{N}$ levels from 40 to $80 \mathrm{~kg} \mathrm{~N}$ fed $^{-1}$ significantly increased top, root and sugar yields and sucrose \%, in the two seasons. Combination of $\mathrm{CM}+80 \mathrm{~kg} \mathrm{~N}^{-1} \mathrm{~d}^{-1}(100 \% \mathrm{~N})$, produced the highest extractable sugar $\%(15.53 \%)$ in the $1^{s t}$ season and increased root yield by $(11.42$ and $3.16 \%$ ), sugar yield by (13.62 and $5.22 \%$ ) in the $1^{s t}$ and $2^{\text {nd }}$ seasons, respectively and sucrose $\%$ by $(2.08 \%)$ in the $1^{\text {st }}$ season, as compared with adding $80 \mathrm{~kg} \mathrm{~N}$ fed $^{-1}(100 \% \mathrm{~N})$ alone. Combination of $\mathrm{CM}+$ $60 \mathrm{~kg} \mathrm{~N} \mathrm{fed}^{-1}$ increased sucrose $\%$ by $(4.13 \%)$ as compared with $80 \mathrm{~kg} \mathrm{~N}$ fed $^{-1}$ alone in the $2^{\text {nd }}$ season. Compost alone gave the highest purity and Alkalinity coefficient (AC) \% and the lowest impurities (K, $\mathrm{Na}$ and Alpha-amino $\mathrm{N} \%$ ) and SLM\% in the $2^{\text {nd }}$ season. Various interaction orders among the two factors affected significantly all traits under study. According to this investigation, to gain high sugar yield of sugar beet, KWS1436 fertilized by 4 tons compost $+\mathrm{N}$ level of $80 \mathrm{~kg} \mathrm{~N} \mathrm{fed}^{-1}(100 \% \mathrm{~N})$ is recommended under saline sandy soil conditions of Wadi El-Natroon and similar areas..
\end{abstract}

Key words: compost, nitrogen fertilizer, sandy soil, sugar beet (Beta vulgaris L.), varieties, yield and quality.

\section{INTRODUCTION}

Sugar beet (Beta vulgaris L.) is grown for sugar production and it is the second source of sugar after sugar cane. Sugar beet has some advantages: the ability to store high sucrose percentage, the byproducts which are used for alcohol production and livestock and the wide adaptability to grow in poor, saline and alkaline soils. Despite the recently introduced sugar beet in Egypt, it is of great importance in newly reclaimed sandy soils at the northern parts of Egypt without competition from other crops because of its tolerance to salinity and the ability to produce high yields of sugar under saline affected soil and water conditions. Under continuous cropping or in the newly reclaimed lands, the soils have short supply of some elements especially nitrogen. In most sugar beet growing regions, $\mathrm{N}$ is the most important fertilizer element for normal growth and high yield of root and sugar. Many investigations have been oriented to optimize the use of nitrogen through a better understanding of crop requirements under varying conditions of soil environmental pollution (Salama and Badawi, 1996; Ghura et al. 2000 and Attallah and El Etreiby 2002). El-Sarag (2009) stated that application of $120 \mathrm{~kg} \mathrm{~N}^{-1}$ gave the heighest root yield (33.15 and 35.22 ton $/ \mathrm{fed}^{-1}$ ) in both 
seasons and increased sugar yield by $21.2,14.1$, $0.37 \%$ compared with 60,80 and $100 \mathrm{~kg} \mathrm{~N}^{-1} \mathrm{fed}^{-1}$. Ferweez et al., (2011) stated that the highest values of root yield (36.38 ton/fed) were recorded by $120 \mathrm{~kg} \mathrm{~N}$ fed $^{-1}$. Adding100 $\mathrm{kg} \mathrm{N}$ fed $^{-1}$ recorded the highest value of sugar yield (4.18 ton/fed) and caused a decrease in pol\% by 5.74 and $16.89 \%$, and $\mathrm{Na}, \mathrm{K}$, and alpha amino-N\% compared with the control $\left(80 \mathrm{~kg} \mathrm{~N} \mathrm{fed}^{-1}\right)$. Also Aboshady et al., (2011) reported that the application of $\mathrm{N}$ - at the rate of $105 \mathrm{~kg} \mathrm{~N} / \mathrm{fad}$. gave the highest root, top and sugar yields as well as $\mathrm{Na}, \mathrm{K}$, alpha amino-N and sugar loss in molasses. While sugar extractable \%, extractability \%and alkalinity coefficient recorded the lowest values.

Recently, some investigators utilized the farmyard manure (FYM) to fertilize sugar beet to decrease the cost and minimize the pollution due to mineral fertilizers. Zalat and Nemeat Alla (2001) found that farmyard manure increased sucrose $\%$ and TSS $\%$ while mineral $\mathrm{N}$ gave the highest root, top and sugar yield. Attallah and El Etreiby (2002) indicated that relative to untreated soil, treatment compost + mineral-N increased root yield, sucrose $\%$ and TSS $\%$ by $87.40,15.71$ and $15.73 \%$, respectively. Also, Marinkovic et al. (2004) found that the application of organic fertilizer increased root yield from 1.41 to 2.13 ton/ha. compared with the untreated treatment. Similarly, Hassan (2004) reported that the application of organic fertilizer increased the root yield, sugar yield, sucrose content and purity\%. Mohamed (2008) revealed that compost significantly increased sucrose $\%$ and sugar yield compared to using mineral-N fertilizers. Compost has been shown to have a positive effect on agricultural soils and crop production, because compost provides a whole array of nutrients for the soil (Seok-In and Hee-Myong, 2009). Moreover, its use reduces the dependence on mineral fertilizers and contributes to pollution free environment, which is of great need (Attallah et al, 1997; Attallah and El Etreiby 2002 and SeokIn and Hee-Myong 2009).

Concerning varieties, Ali (2000) tested three sugar beet varieties Pleno, Kawemira and Lola. He found that Lola surpassed the other varieties in root and sugar yields. Badawi et al. (2002) evaluated four sugar beet cultivars i.e., Top, Lola, Pleno and Kawemira. They found that, Kawemira was superior in sucrose\%, root, top and sugar yields. Azzazy et al. (2007) and El-Sheikh et al. (2009) showed that the evaluated sugar beet varieties varied significantly in root and sugar yields, while sucrose and purity \% did not differ significantly. Sugar beet variety KWS-9422 gave the highest root and sugar yields. Ouda (2009) tested two sugar beet varieties (Athos poly and Lados) and found that, Lodos variety surpassed Athos Poly in top, root and sugar yields. On the other hand, Omar (2007) indicated that sugar beet varieties had no significant effect on root and sugar yields in two seasons.

The main objectives of the present research were to find out the best variety to be grown under the stress conditions (sandy soil and salinity irrigation water of 2496-2650 ppm) and the optimum nitrogen level with organic fertilizer to obtain the highest yield and quality of sugar beet.

\section{MATERIALS AND METHODS}

Two field experiments were carried out at the Agricultural Experiments Desert Station of the Faculty of Agriculture, Cairo University in Wadi El-Natroon, El-Beheira Governorate, during the two successive winter seasons of 2008/2009 and $2009 / 2010$ to study the response of three sugar beet varieties (KWS1436, Swello and Faraha) to five treatments of compost (CM), and of Mineral$\mathrm{N}$ fertilizer and their combination: 4 tons $\mathrm{fed}^{-1}$ of (CM), 4 tons $\mathrm{fed}^{-1}$ of $(\mathrm{CM})$ and $80 \mathrm{~kg} \mathrm{~N}$ fed ${ }^{1}(100 \% \mathrm{~N}), 4$ tons $^{-1} \mathrm{fed}^{-1}$ of $(\mathrm{CM})$ and $60 \mathrm{~kg} \mathrm{~N}^{-} \mathrm{fed}^{-}$ ${ }^{1}(75 \% \mathrm{~N}), 4$ tons $\mathrm{fed}^{-1}$ of $(\mathrm{CM})$ and $40 \mathrm{~kg} \mathrm{~N} \mathrm{fed}^{-1}$ $(50 \% \mathrm{~N})$ and $80 \mathrm{~kg} \quad \mathrm{~N} \quad$ fed $^{-1}(100 \% \quad \mathrm{~N}$, recommended rate) on yield and quality of sugar beet. Treatments were arranged in a split-plot in a randomized complete block design with three replications. The main plots were devoted to the sugar beet varieties, while the sub plots were occupied by fertilizer treatments. Plot area was 21 $\mathrm{m}^{2}$ (6 ridges, $7 \mathrm{~m}$ long and $50 \mathrm{~cm}$ apart). Sugar beet was sown on 10 and 15 October in the two seasons, respectively. All plots were fertilized with $30 \mathrm{~kg} \mathrm{P}_{2} \mathrm{O}_{5}$ /fed before planting in the form of single super-phosphate $\left(15.5 \% \mathrm{P}_{2} \mathrm{O}_{5}\right) .50 \mathrm{~kg}$ $\mathrm{K}_{2} \mathrm{O}$ fed $^{-1}$ in the form of potassium sulphate $(48 \%$ $\mathrm{K}_{2} \mathrm{O}$ ) was added through six equal doses. The first dose was added after thinning and the rest doses were applied at 7-day intervals. Nitrogen fertilizer was applied at levels of 40,60 and $80 \mathrm{~kg} \mathrm{~N}^{\mathrm{fed}}{ }^{-1}$, in the form of ammonium nitrate $(33.5 \% \mathrm{~N})$ in six equal doses; the first dose was added after thinning and the rest doses were applied at 7-day intervals. 2 ton/Fed. of compost (CM) was broadcasted on soil two weeks before sowing. All suitable agricultural practices were conducted in the proper time. The mechanical and chemical analyses of the soil, water and compost analysis were carried out by the Reclamation and Development Center for desert soils, Faculty of 
Agriculture, Cairo University (Tables 1, 2 and 3). The two field experiments were conducted under drip irrigation system.

\subsection{Studied characters}

Sugar beet was harvested by hand after 210 days from sowing. Weight per plot was obtained and used to calculate root yield per-feddan. Plants of each plot for various treatments were uprooted and topped to estimate the following characters:

- Yield components:

2.1.1.Average root fresh weight $(\mathrm{kg} / \mathrm{plant})$

2.1.2. Top yield (ton $\mathrm{fed}^{-1}$ )

2.1.3. Root yield (ton fed ${ }^{-1}$ )

2.1.4. Sugar yield (ton $\mathrm{fed}^{-1}$ ) was calculated according to the following equation:

Sugar yield $\left(\right.$ ton $\left.\mathrm{fed}^{-1}\right)=$ Root yield $\left(\right.$ ton $\left.\mathrm{fed}^{-1}\right) \mathrm{X}$ Sugar \%

\subsection{Juice quality}

2.1.1. Sucrose $\%$ was determined as described by Le Docte (1927).

2.2.2. Juice impurity (Potassium \%, Sodium \% and alpha-amino-N \%), Na, K determined using Flame photometer as described by Page (1982) and alpha-amino-N determined using Hydrindnation method according to Carruthers et al. (1962).

2.2.3. Juice purity $\%$ and sugar lost to molasses $\%$ were calculated by the equation of Devillers (1988) as follows:

- Juice purity $\%=99.36-[14.27(\mathrm{~V} 1+\mathrm{V} 2+\mathrm{V} 3) / \mathrm{V} 4]$.

- Sugar loss to molasses \% (SLM) $=0.14$ $(\mathrm{V} 1+\mathrm{V} 2)+0.25(\mathrm{~V} 3)+0.50$

2.2.4. Extractable sugar $\%$ and alkalinity coefficient \% (AC) were calculated as proposed by Dexter et al. (1967):

- Extractable sugar $\%=\mathrm{V} 4-\mathrm{SLM}-0.6$.

- Alkalinity coefficient $(\mathrm{AC})=\mathrm{V} 1+\mathrm{V} 2 / \mathrm{V} 3$.

Where: $\mathrm{V} 1=$ Sodium $\%, \mathrm{~V} 2=$ potassium $\%, \mathrm{~V} 3=$ Alpha-amino-N \% and V4 $=$ Sucrose \%.

Data obtained from each season of the study were statistically analyzed according to procedures outlined by Gomez and Gomez (1984) using M-STAT-C computer program (Freed et al., 1989). The differences among treatment means were compared by Least Significant Differences test (L.S.D) at 0.05 level of propability.

\section{RESULTS AND DISCUSSION \\ 3.1. Effect of varieties}

Data in Table (4) cleared that the evaluated sugar beet varieties differed significantly in root fresh weight, top, root and sugar yields and sucrose \% in both seasons except sugar yield in the $2^{\text {nd }}$ season. KWS1436 sugar beet variety surpassed the other two varieties, Swello and
Faraha in sucrose $\%$ and sugar yield in the two seasons. It recorded $18.27 \%$ and 5.26 ton $\mathrm{fed}^{-1}$, in the $1^{\text {st }}$ season corresponding to $18.07 \%$ and 5.30 ton fed ${ }^{-1}$ in the $2^{\text {nd }}$ one, respectively.

It also produced the highest root yield (28.81 ton $\left.\mathrm{fed}^{-1}\right)$, in the $1^{\text {st }}$ season, Swello variety recorded the highest insignificant root yield (29.96 ton $\mathrm{fed}^{-1}$ ) in the $2^{\text {nd }}$ season.

The variation between varieties is probably due to genetic differences. El-Hinnawy et al. (2003) showed that the genotypes significantly differed in root and sugar yields. Also, Ouda (2009) evaluated two sugar beet varieties (Athos poly and Lodos) and reported that, Lodos variety surpassed Athos Poly in top, root and sugar yields.

Results in Table (5) showed that, all quality parameters i.e. juice impurities $(\mathrm{K}, \mathrm{Na}$ and alphaamino $\mathrm{N} \%$ ), juice purity $\%$, sugar loss to molasses (SLM \%), extractable sugar \% and AC $\%$ were significantly affected by the studied sugar beet varieties in both seasons except for, purity $\%$ and SLM $\%$ in the $2^{\text {nd }}$ season.

KWS1436 variety recorded the highest extractable sugar (15.70 and $15.41 \%)$, and the lowest values of $\mathrm{Na} \%(1.76$ and $1.74 \%$,) in the $1^{s t}$ and $2^{\text {nd }}$ seasons, respectively and recorded the highest purity $(91.89 \%)$ and lowest K $(6.33 \%)$ and SLM\% (1.98\%) in the $1^{s t}$ season. While, Faraha variety was superior to the other two varieties in juice purity $(91.46 \%)$ in the $2^{\text {nd }}$ season. In the same line, Abou El Seoud et al. (2009) recorded significant differences in quality parameters, except for purity and extractable sugar $\%$ among sugar beet varieties.

\subsection{Effects of fertilizer treatments}

The obtained data in Table (6) revealed that combination of $\mathrm{CM}+80 \mathrm{~kg} \mathrm{~N}^{-1} \mathrm{fe}^{-1}(100 \% \mathrm{~N})$, was more effective and produced the highest top yield (13.42 and 15.24 ton $\mathrm{fed}^{-1}$ ), and increased root yield by (11.42 and $3.16 \%)$, sugar yield by (13.62 and $5.22 \%$ ) in the $1^{s t}$ and $2^{\text {nd }}$ seasons, respectively and increased sucrose $\%$ by $(2.08 \%)$ in the $1^{s t}$ season, as compared with adding $80 \mathrm{~kg} \mathrm{~N}^{-}$fed $^{-}$ ${ }^{1}(100 \% \mathrm{~N})$ alone (Table 6). However, using (CM) $+60 \mathrm{~kg} \mathrm{~N} \mathrm{fed}{ }^{-1}(75 \% \mathrm{~N})$ exhibited significant increase in sucrose \% over fertilizing by $80 \mathrm{~kg} \mathrm{~N}$ fed $^{-1}(100 \% \mathrm{~N})$ alone by $4.13 \%$ in the $2^{\text {nd }}$ season. It is important to notice that the differences between $(\mathrm{CM})+60 \mathrm{~kg} \mathrm{~N}^{-1} \mathrm{fe}^{-1}$ and $(\mathrm{CM})+40 \mathrm{~kg} \mathrm{~N}$ $\mathrm{fed}^{-1}(50 \% \mathrm{~N})$ were insignificant for root and sugar yields and sucrose $\%$ in the $1^{\text {st }}$ season.

These results may be due to that compost with high organic matter and low concentration of inorganic and organic pollutants allowed improvements of physical, chemical and 
S. A. Safina and E. M. Abdel Fatah.

Table (1): Physical and chemical properties of soil in 2008/2009and 2009/2010 seasons.

\begin{tabular}{|c|c|c|}
\hline \multirow{2}{*}{ Soil properties } & \multicolumn{2}{|c|}{ Seasons } \\
\hline & $2008 / 2009$ & $2009 / 2010$ \\
\hline \multicolumn{3}{|c|}{ Physical properties } \\
\hline Sand \% & 93.00 & 92.25 \\
\hline Silt \% & 4.56 & 5.19 \\
\hline Clay \% & 2.44 & 2.56 \\
\hline Texture & Sandy & Sandy \\
\hline \multicolumn{3}{|c|}{ Chemical properties } \\
\hline Soil (pH) & 7.81 & 7.75 \\
\hline Ec $(d s / m)$ & 7.80 & 7.50 \\
\hline Organic Matter (\%) & 0.29 & 0.32 \\
\hline Total $\mathrm{CaCo3}(\%)$ & 2.59 & 2.65 \\
\hline Total N (\%) & 0.60 & 0.65 \\
\hline \multicolumn{3}{|c|}{ Soluble anions concentration $(\mathrm{meq} / \mathrm{L})(\mathrm{meq} / \mathrm{100g}$ soil $)$} \\
\hline $\mathrm{Cl}^{-}$ & 77.75 & 77.0 \\
\hline $\mathrm{HCO}_{3}^{-}$ & 0.51 & 0.55 \\
\hline $\mathrm{SO}_{4}^{-}$ & 0.52 & 0.49 \\
\hline \multicolumn{3}{|c|}{ Soluble cations concentration (meq/L) (meq/100g soil) } \\
\hline $\mathrm{Na}^{+}$ & 52.0 & 50.0 \\
\hline $\mathbf{K}^{+}$ & 1.00 & 1.20 \\
\hline $\mathrm{Ca}^{+}$ & 17.00 & 7.50 \\
\hline $\mathrm{Mg}^{+}$ & 17.00 & 18.00 \\
\hline
\end{tabular}

Table (2): Chemical analysis of water samples in 2009 and 2010 years.

\begin{tabular}{|c|c|c|c|c|c|c|c|c|c|c|}
\hline \multirow{2}{*}{ Year } & $\mathbf{p H}$ & \multicolumn{2}{|c|}{$\mathbf{E C}$} & \multicolumn{6}{|c|}{ Ions concentration meq/L } \\
\cline { 2 - 12 } & Unit & $\mathbf{d s} / \mathbf{m}$ & $\mathbf{p p m}$ & $\mathbf{H C O}_{\mathbf{3}}{ }^{-}$ & $\mathbf{C l}^{-}$ & $\mathbf{S O}_{\mathbf{4}}{ }^{-}$ & $\mathbf{C a}^{++}$ & $\mathbf{M g}^{++}$ & $\mathbf{N a}^{+}$ & $\mathbf{K a}^{+}$ \\
\hline $\mathbf{2 0 0 8}$ & 7.49 & 3.9 & 2496 & 3.7 & 31.5 & 7.60 & 4.5 & 5.10 & 34.9 & 0.50 \\
\hline $\mathbf{2 0 0 9}$ & 7.43 & 4.15 & 2656 & 3.2 & 30.0 & 7.10 & 5.0 & 4.0 & 30.0 & 0.42 \\
\hline
\end{tabular}

Table (3): The mean value of chemical composition and DTPA-extractable micronutrients of the used compost.

\begin{tabular}{|c|c|c|c|c|c|c|c|c|c|c|c|c|}
\hline $\begin{array}{c}\text { Ec } \\
\mathbf{D s m}^{-1}\end{array}$ & $\mathbf{p H}$ & $\begin{array}{c}\text { O.C } \\
\text { \% }\end{array}$ & $\begin{array}{c}\text { T.N. } \\
\text { \% }\end{array}$ & $\mathbf{P} \%$ & $\mathbf{K} \%$ & $\begin{array}{c}\text { C/N } \\
\text { Ratio }\end{array}$ & $\begin{array}{c}\text { Ash } \\
\text { \% }\end{array}$ & OM \% & Fe & Zn & Mn & Cu \\
\hline 1.90 & 7.2 & 19.1 & 1.40 & 0.30 & 0.98 & 13.64 & 80.2 & 32.65 & 45.9 & \multicolumn{4}{|c|}{$\left(\mathbf{M g ~ k g}^{-1}\right)$} \\
\hline
\end{tabular}

Table (4): Mean performance of three sugar beet cultivars for top, root and sugar yields and sucrose \% in 2008/2009 and 2009/2010 seasons.

\begin{tabular}{|c|c|c|c|c|}
\hline Variety & $\begin{array}{l}\text { Top yield } \\
\text { (ton fed }^{-1} \text { ) }\end{array}$ & $\begin{array}{c}\text { Root yield (ton } \\
\text { fed }^{-1} \text { ) }\end{array}$ & $\begin{array}{c}\text { Sugar yield } \\
\left.\text { (ton fed }^{-1}\right)\end{array}$ & $\begin{array}{c}\text { Sucrose } \\
\%\end{array}$ \\
\hline \multicolumn{5}{|c|}{$2008 / 2009$} \\
\hline KWS1436 & 12.03 & 28.81 & 5.26 & 18.27 \\
\hline Swello & 12.51 & 26.88 & 4.72 & 17.58 \\
\hline Faraha & 11.32 & 26.47 & 4.74 & 17.84 \\
\hline $\mathrm{LSD}_{0.05}$ & 0.70 & 2.20 & 0.39 & 0.18 \\
\hline \multicolumn{5}{|c|}{$2009 / 2010$} \\
\hline KWS1436 & 11.13 & 29.30 & 5.30 & 18.07 \\
\hline Swello & 12.69 & 29.96 & 5.29 & 17.69 \\
\hline Faraha & 13.44 & 28.31 & 5.22 & 17.96 \\
\hline $\operatorname{LSD}_{0.05}$ & 2.19 & 0.79 & N.S. & 0.27 \\
\hline
\end{tabular}


Table (5): Mean performance of three sugar beet cultivars for root juice quality percentages at harvest in 2008/2009 and 2009/2010 seasons.

\begin{tabular}{|c|c|c|c|c|c|c|c|}
\hline \multirow[b]{2}{*}{ Variety } & \multicolumn{3}{|c|}{ Juice impurities \% } & \multirow[b]{2}{*}{$\begin{array}{c}\text { Juice } \\
\text { purity \% }\end{array}$} & \multirow{2}{*}{$\begin{array}{c}\text { Sugar loss } \\
\text { to } \\
\text { molasses } \\
\text { (SLM \%) }\end{array}$} & \multirow[b]{2}{*}{$\begin{array}{c}\text { Extr. } \\
\text { sugar \% }\end{array}$} & \multirow{2}{*}{$\begin{array}{c}\text { Alkalinity } \\
\text { coeff. } \\
\text { (AC \%) }\end{array}$} \\
\hline & $\begin{array}{l}\mathrm{K} \\
\%\end{array}$ & $\begin{array}{c}\mathrm{Na} \\
\%\end{array}$ & $\begin{array}{c}\text { Alpha- } \\
\text { amino-N } \\
\%\end{array}$ & & & & \\
\hline \multicolumn{8}{|c|}{$2008 / 2009$} \\
\hline KWS1436 & 6.33 & 1.76 & 1.36 & 91.89 & 1.98 & 15.70 & 6.09 \\
\hline Swello & 6.64 & 1.77 & 1.30 & 91.46 & 2.00 & 14.98 & 6.61 \\
\hline Faraha & 6.50 & 1.79 & 1.35 & 91.62 & 2.00 & 15.24 & 6.29 \\
\hline $\mathrm{LSD}_{0.05}$ & 0.06 & 0.02 & 0.06 & 0.12 & 0.01 & 0.18 & 0.34 \\
\hline \multicolumn{8}{|c|}{$\frac{1}{2009 / 2010}$} \\
\hline KWS1436 & 6.97 & 1.74 & 1.40 & 91.38 & 2.07 & 15.41 & 6.51 \\
\hline Swello & 6.57 & 1.81 & 1.48 & 91.35 & 2.04 & 15.05 & 5.87 \\
\hline Faraha & 6.66 & 1.86 & 1.44 & 91.46 & 2.06 & 15.30 & 6.01 \\
\hline $\operatorname{LSD}_{0.05}$ & 0.30 & 0.06 & 0.01 & N.S. & N.S. & 0.27 & 0.27 \\
\hline
\end{tabular}

N.S. = not significant

Table (6): Effect of fertilizer treatments on top, root and sugar yields (ton fed $^{-1}$ ) and sucrose $\%$ in $2008 / 2009$ and $2009 / 2010$ seasons.

\begin{tabular}{|c|c|c|c|c|}
\hline $\begin{array}{r}\text { Fertilizer } \\
\text { treat } \\
\text { ment } \\
\text { s }\end{array}$ & $\begin{array}{l}\text { Top yield } \\
\text { (ton fed }^{-1} \text { ) }\end{array}$ & $\begin{array}{c}\text { Root yield } \\
\text { (ton fed }^{-1} \text { ) }\end{array}$ & $\begin{array}{c}\text { Sugar yield } \\
{\left.\text { (ton } \text { fed }^{-1}\right)}^{\text {a }}\end{array}$ & $\begin{array}{c}\text { Sucrose } \\
\%\end{array}$ \\
\hline \multicolumn{5}{|c|}{$2008 / 2009$} \\
\hline Compost (CM) & 9.76 & 25.01 & 4.48 & 17.93 \\
\hline $\mathrm{CM}+80 \mathrm{~kg} \mathrm{~N}$ & 13.42 & 30.74 & 5.59 & 18.18 \\
\hline $\mathrm{CM}+60 \mathrm{~kg} \mathrm{~N}$ & 12.42 & 26.62 & 4.77 & 17.93 \\
\hline $\mathrm{CM}+40 \mathrm{~kg} \mathrm{~N}$ & 11.18 & 26.95 & 4.76 & 17.63 \\
\hline $80 \mathrm{~kg} \mathrm{~N}$ & 13.00 & 27.59 & 4.92 & 17.81 \\
\hline $\mathrm{LSD}_{0.05}$ & 0.51 & 1.23 & 0.23 & 0.34 \\
\hline \multicolumn{5}{|c|}{$2009 / 2010$} \\
\hline Compost (CM) & 9.37 & 25.51 & 4.48 & 17.56 \\
\hline $\mathrm{CM}+80 \mathrm{~kg} \mathrm{~N}$ & 15.24 & 31.31 & 5.64 & 18.02 \\
\hline $\mathrm{CM}+60 \mathrm{~kg} \mathrm{~N}$ & 13.07 & 30.00 & 5.52 & 18.40 \\
\hline $\mathrm{CM}+40 \mathrm{~kg} \mathrm{~N}$ & 11.67 & 28.78 & 5.34 & 17.89 \\
\hline $80 \mathrm{~kg} \mathrm{~N}$ & 12.76 & 30.35 & 5.36 & 17.67 \\
\hline $\mathrm{LSD}_{0.05}$ & 0.75 & 0.85 & 0.15 & 0.12 \\
\hline
\end{tabular}

biochemical characteristics of the soil and encouraged plant uptake of $\mathrm{N}$ and other elements and activated accumulation of carbohydrates, which in turn enhanced sugar beet root fresh weight, yield productivity and quality.

In general, in combination treatments increasing $\mathrm{N}$ levels from $40 \mathrm{~kg} \mathrm{~N}$ fed $^{-1}(50 \% \mathrm{~N})$ to
$80 \mathrm{~kg} \mathrm{~N} \mathrm{fed}{ }^{-1}(100 \% \mathrm{~N})$ significantly increased root fresh weight, top, root and sugar yields and sucrose in both seasons, which can be explained by the role of nitrogen in enhancing growth, chlorophyll formation and photosynthesis process. Similar results were reported by many investigators in other sugar beet production areas 
Table (7):Effect of fertilizer treatments on impurity \%, purity \%, sugar loss to molasses, extractable sugar \% and alkalinity coeff. $\%$ in $2008 / 2009$ and $2009 / 2010$ seasons.

\begin{tabular}{|c|c|c|c|c|c|c|c|}
\hline \multirow[b]{2}{*}{ Fertilizers } & \multicolumn{3}{|c|}{ Juice impurity \% } & \multirow{2}{*}{$\begin{array}{c}\text { Juice } \\
\text { purity } \\
\%\end{array}$} & \multirow{2}{*}{$\begin{array}{c}\text { Sugar } \\
\text { loss to } \\
\text { molasses } \\
(\text { SLM)\% }\end{array}$} & \multirow{2}{*}{$\begin{array}{c}\text { Extr. } \\
\text { sugar } \\
\%\end{array}$} & \multirow{2}{*}{$\begin{array}{c}\text { Alkalinity } \\
\text { coeff. (AC } \\
\% \text { ) }\end{array}$} \\
\hline & $\begin{array}{l}\mathrm{K} \\
\%\end{array}$ & $\begin{array}{l}\mathrm{Na} \\
\%\end{array}$ & $\begin{array}{c}\text { Alpha- } \\
\text { amino-N } \\
\%\end{array}$ & & & & \\
\hline \multicolumn{8}{|c|}{$2008 / 2009$} \\
\hline Compost (CM) & 6.62 & 1.67 & 1.37 & 91.54 & 2.01 & 15.32 & 6.11 \\
\hline $\mathrm{CM}+80 \mathrm{~kg} \mathrm{~N}$ & 6.66 & 1.92 & 1.36 & 91.49 & 2.05 & 15.53 & 6.41 \\
\hline $\mathrm{CM}+60 \mathrm{~kg} \mathrm{~N}$ & 6.12 & 1.80 & 1.07 & 92.20 & 1.88 & 15.45 & 7.41 \\
\hline $\mathrm{CM}+40 \mathrm{~kg} \mathrm{~N}$ & 6.27 & 1.62 & 1.32 & 92.11 & 1.90 & 15.13 & 5.98 \\
\hline $80 \mathrm{~kg} \mathrm{~N}$ & 6.79 & 1.85 & 1.56 & 90.97 & 2.14 & 15.08 & 5.73 \\
\hline $\mathrm{LSD}_{0.05}$ & 0.07 & 0.03 & 0.03 & 0.19 & 0.01 & 0.21 & 0.23 \\
\hline \multicolumn{8}{|c|}{$2009 / 2010$} \\
\hline Compost (CM) & 5.93 & 1.66 & 1.06 & 92.32 & 1.83 & 15.13 & 7.17 \\
\hline $\mathrm{CM}+80 \mathrm{~kg} \mathrm{~N}$ & 7.43 & 1.83 & 1.61 & 90.70 & 2.20 & 15.21 & 5.82 \\
\hline $\mathrm{CM}+60 \mathrm{~kg} \mathrm{~N}$ & 6.74 & 1.89 & 1.36 & 91.60 & 2.05 & 15.76 & 6.58 \\
\hline $\mathrm{CM}+40 \mathrm{~kg} \mathrm{~N}$ & 6.85 & 1.85 & 1.55 & 91.18 & 2.11 & 15.19 & 5.78 \\
\hline $80 \mathrm{~kg} \mathrm{~N}$ & 6.72 & 1.78 & 1.62 & 91.18 & 2.09 & 14.97 & 5.28 \\
\hline $\mathrm{LSD}_{0.05}$ & 0.24 & 0.05 & 0.06 & 0.24 & 0.04 & 0.13 & 0.34 \\
\hline
\end{tabular}

Table (8): Effect of interaction between sugar beet varieties and fertilization treatments on top yield, root yield and sugar yield in 2008/2009 and 2009/2010 seasons.

\begin{tabular}{|c|c|c|c|c|c|c|c|}
\hline \multirow{2}{*}{ Variety } & \multirow{2}{*}{$\begin{array}{c}\text { Fertilizer } \\
\text { treatments }\end{array}$} & \multicolumn{2}{|c|}{ Top yield (ton fed ${ }^{-1}$ ) } & \multicolumn{2}{|c|}{ Root yield (ton fed $^{-1}$ ) } & \multicolumn{2}{|c|}{ Sugar yield (ton fed $\left.^{-1}\right)$} \\
\hline & & 2009 & 2010 & 2009 & 2010 & 2009 & 2010 \\
\hline \multirow{5}{*}{$\begin{array}{l}\text { KWS- } \\
1436\end{array}$} & Compost (CM) & 8.40 & 8.51 & 25.20 & 25.48 & 4.71 & 4.55 \\
\hline & $\mathrm{CM}+80 \mathrm{~kg} \mathrm{~N}$ & 14.00 & 13.53 & 30.61 & 31.55 & 5.68 & 6.00 \\
\hline & $\mathrm{CM}+60 \mathrm{~kg} \mathrm{~N}$ & 13.13 & 11.20 & 29.10 & 29.73 & 5.06 & 5.23 \\
\hline & $\mathrm{CM}+40 \mathrm{~kg} \mathrm{~N}$ & 11.20 & 11.20 & 29.06 & 29.40 & 5.41 & 5.29 \\
\hline & $80 \mathrm{kgN}$ & 13.44 & 11.20 & 30.05 & 30.35 & 5.44 & 5.44 \\
\hline \multirow{5}{*}{ Swello } & Compost (CM) & 9.80 & 11.20 & 25.20 & 25.85 & 4.37 & 4.78 \\
\hline & $\mathrm{CM}+80 \mathrm{~kg} \mathrm{~N}$ & 14.59 & 14.00 & 30.80 & 31.22 & 5.21 & 5.17 \\
\hline & $\mathrm{CM}+60 \mathrm{~kg} \mathrm{~N}$ & 12.93 & 12.60 & 25.20 & 30.87 & 4.68 & 5.88 \\
\hline & $\mathrm{CM}+40 \mathrm{~kg} \mathrm{~N}$ & 11.23 & 12.60 & 26.60 & 30.80 & 4.52 & 5.34 \\
\hline & $80 \mathrm{kgN}$ & 14.00 & 13.07 & 26.60 & 31.05 & 4.81 & 5.28 \\
\hline \multirow{5}{*}{ Faraha } & Compost (CM) & 11.07 & 8.40 & 24.64 & 25.20 & 4.37 & 4.11 \\
\hline & $\mathrm{CM}+80 \mathrm{~kg} \mathrm{~N}$ & 11.67 & 18.20 & 30.80 & 31.17 & 5.88 & 5.75 \\
\hline & $\mathrm{CM}+60 \mathrm{~kg} \mathrm{~N}$ & 11.20 & 15.40 & 25.57 & 29.40 & 4.57 & 5.46 \\
\hline & $\mathrm{CM}+40 \mathrm{~kg} \mathrm{~N}$ & 11.12 & 11.20 & 25.20 & 26.13 & 4.36 & 5.40 \\
\hline & $80 \mathrm{~kg} \mathrm{~N}$ & 11.57 & 14.00 & 26.13 & 29.64 & 4.49 & 5.36 \\
\hline & $\mathrm{LSD}_{0.05}$ & 0.89 & 1.28 & 2.13 & 1.46 & 0.39 & 0.27 \\
\hline
\end{tabular}

(El-Hinnawy et al., 1998; Attallah and El Etreiby 2002; Attallah 2004; Leilah et al. (2005) and ElGeddawy et al. (2006). They reported that combination of $\mathrm{CM}$ with mineral-N had marked positive effect on root and sugar yields and sucrose $\%$. 
Table (9): Effect of interaction between sugar beet varieties and fertilization treatments on sucrose \%, potassium \%, sodium \% and alpha-amino- $\mathrm{N} \%$ in 2008/2009 and 2009/2010 seasons.

\begin{tabular}{|c|c|c|c|c|c|c|c|c|c|}
\hline \multirow[t]{2}{*}{ Variety } & \multirow{2}{*}{$\begin{array}{l}\text { Fertilizer } \\
\text { treatments }\end{array}$} & \multicolumn{2}{|c|}{ Sucrose \% } & \multicolumn{2}{|c|}{$\begin{array}{c}\text { Potassium }(\mathrm{K}) \\
\%\end{array}$} & \multicolumn{2}{|c|}{ Sodium (Na) \% } & \multicolumn{2}{|c|}{$\begin{array}{c}\text { Alpha-amino-N } \\
\%\end{array}$} \\
\hline & & 2009 & 2010 & 2009 & 2010 & 2009 & 2010 & 2009 & 2010 \\
\hline \multirow{5}{*}{$\begin{array}{l}\text { KWS- } \\
1436\end{array}$} & Compost CM) & 18.68 & 17.85 & 6.10 & 6.52 & 1.69 & 1.71 & 1.26 & 1.10 \\
\hline & $\mathrm{CM}+80 \mathrm{~kg} \mathrm{~N}$ & 18.53 & 19.03 & 6.07 & 7.03 & 2.02 & 1.89 & 1.55 & 1.50 \\
\hline & $\mathrm{CM}+60 \mathrm{~kg} \mathrm{~N}$ & 17.38 & 17.60 & 6.20 & 7.26 & 1.76 & 1.75 & 1.02 & 1.05 \\
\hline & $\mathrm{CM}+40 \mathrm{~kg} \mathrm{~N}$ & 18.60 & 17.98 & 6.13 & 7.09 & 1.58 & 1.65 & 1.49 & 1.57 \\
\hline & $80 \mathrm{~kg} \mathrm{~N}$ & 18.15 & 17.91 & 7.16 & 6.97 & 1.82 & 1.70 & 1.49 & 1.76 \\
\hline \multirow{5}{*}{ Swello } & Compost $\mathrm{CM}$ ) & 17.35 & 18.50 & 6.90 & 5.84 & 1.56 & 1.61 & 1.60 & 0.99 \\
\hline & $\mathrm{CM}+80 \mathrm{~kg} \mathrm{~N}$ & 16.90 & 16.57 & 7.68 & 7.84 & 1.87 & 1.91 & 1.40 & 1.49 \\
\hline & $\mathrm{CM}+60 \mathrm{~kg} \mathrm{~N}$ & 18.57 & 19.02 & 6.11 & 5.79 & 1.80 & 1.96 & 1.06 & 1.51 \\
\hline & $\mathrm{CM}+40 \mathrm{~kg} \mathrm{~N}$ & 17.00 & 17.33 & 6.65 & 6.68 & 1.75 & 1.68 & 1.02 & 1.84 \\
\hline & $80 \mathrm{~kg} \mathrm{~N}$ & 18.08 & 17.02 & 5.87 & 6.71 & 1.83 & 1.92 & 1.43 & 1.57 \\
\hline \multirow{5}{*}{ Faraha } & Compost CM) & 17.75 & 16.32 & 6.85 & 5.43 & 1.77 & 1.65 & 1.26 & 1.10 \\
\hline & $\mathrm{CM}+80 \mathrm{~kg} \mathrm{~N}$ & 19.10 & 18.45 & 6.24 & 7.41 & 1.88 & 1.70 & 1.14 & 1.84 \\
\hline & $\mathrm{CM}+60 \mathrm{~kg} \mathrm{~N}$ & 17.85 & 18.58 & 6.04 & 7.17 & 1.85 & 1.96 & 1.13 & 1.51 \\
\hline & $\mathrm{CM}+40 \mathrm{~kg} \mathrm{~N}$ & 17.30 & 18.37 & 6.04 & 6.79 & 1.54 & 2.23 & 1.46 & 1.25 \\
\hline & $80 \mathrm{~kg} \mathrm{~N}$ & 17.20 & 18.07 & 7.34 & 6.49 & 1.90 & 1.73 & 1.76 & 1.52 \\
\hline \multicolumn{2}{|c|}{$\mathrm{LSD}_{0.05}$} & 0.32 & 0.21 & 0.12 & 0.42 & 0.05 & 0.09 & 0.05 & 0.11 \\
\hline
\end{tabular}

Table (10): Effect of interaction between sugar beet varieties and fertilization treatments on purity \%, sugar losses to molasses $\%$, extractable sugar $\%$ and alkalinity coefficient $\%$ in $2008 / 2009$ and 2009/2010 seasons.

\begin{tabular}{|c|c|c|c|c|c|c|c|c|c|}
\hline \multirow[t]{2}{*}{ Variety } & \multirow{2}{*}{$\begin{array}{l}\text { Fertilizer } \\
\text { treatments }\end{array}$} & \multicolumn{2}{|c|}{ Juice purity \% } & \multicolumn{2}{|c|}{\begin{tabular}{|c|}
$\begin{array}{c}\text { Sugar } \\
\text { losses to molasses } \\
\text { (SLM)\% }\end{array}$ \\
\end{tabular}} & \multicolumn{2}{|c|}{$\begin{array}{c}\text { Extr. } \\
\text { sugar \% }\end{array}$} & \multicolumn{2}{|c|}{$\begin{array}{c}\text { Alkalinity coeff. } \\
(\mathrm{AC} \%)\end{array}$} \\
\hline & & 2009 & 2010 & 2009 & 2010 & 2009 & 2010 & 2009 & 2010 \\
\hline \multirow{5}{*}{$\begin{array}{l}\text { KWS- } \\
1436\end{array}$} & Compost (CM) & 92.11 & 91.90 & 1.91 & 1.93 & 16.18 & 15.32 & 6.21 & 7.51 \\
\hline & $\mathrm{CM}+80 \mathrm{~kg} \mathrm{~N}$ & 91.94 & 91.55 & 2.02 & 2.12 & 15.92 & 16.30 & 5.22 & 5.96 \\
\hline & $\mathrm{CM}+60 \mathrm{~kg} \mathrm{~N}$ & 91.99 & 91.20 & 1.87 & 2.02 & 14.91 & 14.98 & 7.83 & 8.56 \\
\hline & $\mathrm{CM}+40 \mathrm{~kg} \mathrm{~N}$ & 92.30 & 91.18 & 1.95 & 2.12 & 16.05 & 15.27 & 5.17 & 5.59 \\
\hline & $80 \mathrm{~kg} \mathrm{~N}$ & 91.12 & 91.05 & 2.13 & 2.15 & 15.42 & 15.16 & 6.02 & 4.93 \\
\hline \multirow{5}{*}{ Swello } & Compost $(\mathrm{CM})$ & 91.08 & 92.86 & 2.09 & 1.79 & 14.66 & 16.11 & 5.28 & 7.58 \\
\hline & $\mathrm{CM}+80 \mathrm{~kg} \mathrm{~N}$ & 90.12 & 89.67 & 2.19 & 2.24 & 14.11 & 13.73 & 6.84 & 6.56 \\
\hline & $\mathrm{CM}+60 \mathrm{~kg} \mathrm{~N}$ & 92.46 & 92.42 & 1.87 & 1.96 & 16.09 & 16.47 & 7.45 & 5.15 \\
\hline & $\mathrm{CM}+40 \mathrm{~kg} \mathrm{~N}$ & 92.11 & 90.97 & 1.82 & 2.13 & 14.58 & 14.60 & 7.57 & 4.54 \\
\hline & $80 \mathrm{~kg} \mathrm{~N}$ & 91.54 & 90.82 & 2.05 & 2.10 & 15.44 & 14.32 & 5.92 & 5.50 \\
\hline \multirow{5}{*}{ Faraha } & Compost (CM) & 91.42 & 92.20 & 2.02 & 1.77 & 15.13 & 13.95 & 6.85 & 6.43 \\
\hline & $\mathrm{CM}+80 \mathrm{~kg} \mathrm{~N}$ & 92.40 & 90.89 & 1.93 & 2.24 & 16.57 & 15.61 & 7.17 & 4.95 \\
\hline & $\mathrm{CM}+60 \mathrm{~kg} \mathrm{~N}$ & 92.14 & 91.19 & 1.89 & 2.16 & 15.36 & 15.83 & 6.96 & 6.04 \\
\hline & $\mathrm{CM}+40 \mathrm{~kg} \mathrm{~N}$ & 91.91 & 91.38 & 1.93 & 2.08 & 14.77 & 15.69 & 5.21 & 7.22 \\
\hline & $80 \mathrm{~kg} \mathrm{~N}$ & 90.24 & 91.66 & 2.23 & 2.03 & 14.37 & 15.44 & 5.25 & 5.41 \\
\hline \multicolumn{2}{|c|}{$\mathrm{LSD}_{0.05}$} & 0.33 & 0.42 & 0.02 & 0.08 & 0.34 & 0.23 & 0.39 & 0.59 \\
\hline
\end{tabular}

Data shown in Table (7) indicated that the use compost, Mineral- $\mathrm{N}$ and their combination had a significant effect on juice impurities (K, Na and alpha-amino $\mathrm{N} \%$ ), juice purity $\%$, sugar loss to molasses (SLM \%), extractable sugar \% and AC $\%$. In combined treatments, increasing $\mathrm{N}$ levels from $40 \mathrm{~kg} \mathrm{~N} \mathrm{fed}{ }^{-1}(50 \% \mathrm{~N})$ to $80 \mathrm{~kg} \mathrm{~N}^{-}$ ${ }^{1}(100 \% \mathrm{~N})$ significantly increased, $\mathrm{K}$, alpha-amino $\mathrm{N} \%$ and SLM\% in the two seasons and $\mathrm{Na} \%$ in the $1^{\text {st }}$ season and decreased purity $\%$. This effect may be due to the role of high $\mathrm{N}$ level that stimulates vegetative growth and hence more 
essential elements absorbed, in addition to the role of organic matter (compost) in increasing soil microbes and release the available nutrients which increased its rate in beet root at harvest and increased non -sugar component and sugar loss to molasses \% (Abou El Seoud et al., 2009). These results are in harmony with those obtained by Moustafa et al. (2005) and Abou El-Fotoh and Abou El-Magd (2006).

In the $1^{\text {st }}$ season (Table 7), the combination of $\mathrm{CM}+60 \mathrm{~kg} \mathrm{~N}^{-1} \mathrm{fe}^{-1}(75 \% \mathrm{~N})$, was more effective and recorded the highest values of purity and AC $\%$ (92.20 and $7.41 \%$, respectively) as well as the lowest K\% (6.12\%), alpha-amino N \% ( $1.07 \%)$ and SLM \% $(1.88 \%)$. However, in the $2^{\text {nd }}$ season compost alone gave the highest purity and AC \% (92.32 and $7.17 \%$, respectively) and the lowest impurities percentages of $\mathrm{K}, \mathrm{Na}$ and alpha-amino $\mathrm{N} \%$ (5.93, 1.66 and $1.06 \%$, respectively) and SLM \% (1.83\%).

The increase of purity $\%$ and reduction of SLM may be attributed to decrease of non- sugar component, which necessarily had been taken into account of almost calculated aimed to assessing the contribution of the non - sugar to potential loss of sugar as mentioned before. From data in Table (7) it can be noticed that, extractable sugar $\%$ recorded the highest values $(15.53 \%$ and 15.76 $\%$,) by using $\mathrm{CM}+80 \mathrm{~kg} \mathrm{~N}^{-1} \mathrm{de}^{-1}(100 \% \mathrm{~N})$ in the $1^{\text {st }}$ season and by using $\mathrm{CM}+60 \mathrm{~kg} \mathrm{~N}^{-1} \mathrm{fed}^{-1}(75 \%$ $\mathrm{N}$ ) in the $2^{\text {nd }}$ season. Such effect was compensated by corresponding apparent increase in sucrose $\%$ as reported before in Table (6).

\subsection{Interaction effects}

Interaction among varieties and fertilizer treatment affected significantly all traits under study (Tables 8, 9 and 10). In the $1^{\text {st }}$ season, root and sugar yields and sucrose $\%$ of Faraha variety fertilized by $\mathrm{CM}+80 \mathrm{~kg} \mathrm{~N}^{-1} \mathrm{fed}^{-1}$ recorded the highest values ( 30.80 ton $\mathrm{fed}^{-1}, 5.88$ ton fed $^{-1}$ and $19.10 \%$, respectively) (Tables 8 and 9). While, fertilizing variety Swello by $\mathrm{CM}+60 \mathrm{~kg} \mathrm{~N}^{-1} \mathrm{~d}^{-1}$ gave the highest value of purity \% (92.46\%) (Table 10).

In the $2^{\text {nd }}$ season, the highest values of root and sugar yields (31.55 and 6.00 ton $\mathrm{fed}^{-1}$ ) and sucrose $\%(19.03 \%)$ were obtained by KWS1436 variety fertilized by $\mathrm{CM}+80 \mathrm{~kg} \mathrm{~N}^{-1} \mathrm{fed}^{-1}$ (Tables 8 and 9). Fertilizing Faraha variety by compost alone recorded the lowest root and sugar yields (24.64, 25.20 and $4.36,4.11$ ton $\mathrm{fed}^{-1}$, respectively) in the $1^{\text {st }}$ and $2^{\text {nd }}$ seasons, respectively (Table 8 ).

From these results, it could be concluded that fertilizing sugar beet varieties KWS1436 and Faraha by 4 ton compost $+80 \mathrm{~kg} \mathrm{~N}$ fed $^{-1}(100 \%$
$\mathrm{N}$ ), could be recommended to gain high sugar yield under saline sandy soil condition of Wadi El-Natroon and similar areas.

\section{REFERENCES}

Aboshady, Kh. A., Zalat S.S. and Ibraheim M. F. M. (2011). Influence of use nitrogen fertilizer levels and sources for late sowing date on yield and quality of sugar beet (Beta vulgaris L.) in North Nile Delta . Plant Production Mansoura Univ., 2(3): 423-435.

Abou El-Fotoh H.G. and Abou EL- Magd B.M. (2006). Sugar beet productivity and quality as affected by N sources and rates. Egypt J. Appl. Sci., 21 (5): 375-386.

Abou El- Seoud I.I.A., Badr Elham A. and Elshimaa A. E. (2009). Response of two sugar beet varieties to chicken manure and phosphorine application. Alex. Sci. Exch. J., 30 (4): 433-444.

Ali I.F.A. (2000). Factors affecting yield of some sugar beet varieties in newly reclaimed soils. M. Sc. Thesis, Fac. Agric., Cairo Univ., Egypt.

Attallah M.Z. (2004). Effect of biofertilizer, city garbage compost , mineral-N and their combinations on productivity of sugar beet cultivars. J Agric. Sci. Mansoura Univ., 29(7): 4281-4294.

Attallah M.Z. and El Etreiby F. (2002). The effect of compost and mineral- $\mathrm{N}$ on soil properties, ten sugar beet varieties and nutrient contents. Alex. Sci. Exch., 23 (1): 109-120.

Attallah M.Z., El-Deeb M.H., Younan N.Z. and Ghura N.S. (1997). Response of eight sugar beet varieties to city garbage compost in combination with chemical fertilizer. J. Agric. Sci. Mansoura Univ., 22 (3): 941-950.

Azzazy N.B., Shalaby N.M.S. and Abd El-Razek A.M. (2007). Effect of planting density and days to harvest on yield and quality of some sugar beet varieties under Fayoum Governorate conditions. Egypt J.of Appl. Sci., 22 (12A): 101-114.

Badawi M.A., El-Moursy S.A., Mohamed Z.A. and Arafa A.A. (2002). Performance of some sugar beet Beta vulgaris, L. cultivars to planting dates. Pro. Minia $1^{\text {st }}$ Conf. for Agric. and Environ. Sci., Minia, Egypt, March 25-28.

Carruthers A., Oldfield J.F.T. and Teague H.J. (1962). Assessment of beet quality. Paper presented to the $15^{\text {th }}$ Annual Technical Conf. British Sugar Corporation, LTD. 36 pp. (C.F. Sugar beet Crop Book).

Devillers P. (1988). Prevision du sucre melasse. Sucrerie francaise, 129: 190-200. (C.F. Sugar 
Beet Crop Book).

Dexter S.T., Frankes M. G. and Snyder F.W. (1967). A rapid and practical method of determining extractable white sugar as may be applied to the evaluation of agronomic practices and grower deliveries in the sugar beet industry. J. Am. Soc., Sugar beet Technol., 14: 433-454.

El-Geddawy I.H., El-Shafai A.M.A. and Azzazy A.B. (2006). Yield and quality of some sugar beet varieties as affected by planting densities and nitrogen fertilization. J. Agric. Sci. Mansoura Univ., 31(1): 43-54.

El-Hennawy H.H, Ramadan B.S.H. and Mahmoud E.A. (1998). Response of sugar beet to nitrogen fertilization and its time of application. J. Agric. Sci., Mansoura Univ., 23 (3): 969-979.

El-Hinnawy H. H., Ahmed E. A., Ramadan B. S. H., Farag M. A., Al-Jabawi E. M., Mahmoud M.R., Baron P. and Bayoumi M.R. (2003). Variety $\mathrm{x}$ environmental interaction in sugar beet trials. Proc. Int. Conf. on Arab Region and Africa in the world sugar context. Aswan, Egypt 9-12 March.

El-Sarag E.I. (2009). Maximizing sugar beet yield, quality and water use efficiency using some agricultural practices under North Sinai conditions. Bull. Fac. Agric. Cairo Univ. 60: 155-167.

El-Sheikh S.R.E., Khaled K.A.M. and Enan S.A.A.M. (2009). Evaluation of some sugar beet varieties under three harvesting dates. J. Agric. Sci., Mansoura Univ., 34 (3): 15591567.

Ferweez H. Ibrahim M. F. M. and Allan A. M. (2011). Improving yield and quality of sugar beet using boron at different levels of nitrogen fertilizer. Alex. Sci., Exch., 32(1) 51-57.

Freed R., Einensmith S.P., Gutez S., Reicosky D., Smail V.W. and Wolberg P. (1989) Guide to MSTAT-C Analysis of Agronomic Research Experiments. Michigan Univ. East Lansing, U.S.A.

Ghura N.S., Attallah M.Z. and Amer M. (2000). Effect of NPK treatments on yield differential gene action and chemical compostion of three sugar beet varieties. Alex. Sci. Exch., 21 (4): 293-310.

Gomez K.A. and Gomez A.A. (1984). "Statistical procedures for Agricultural Research". $2^{\text {nd }}$ ed. John Willey and Sons. New York, USA.
Hassan W. M. (2004). Effect of some organic fertilizers and sulphur application on yield, quality and nutrient contents of sugar beet. J. Adv. Agric. Res., 10(4): 965-977.

Le-Docte A. (1927). Commercial determination of sugar in the beet root using the Sacks- LeDocte process. Int. Sug. J., 29: 488-492.

Leilah A.A., Badawi M.A. and Said E.M (2005). Effect of planting dates, plant population and nitrogen fertilization on sugar beet productivity under the newly reclaimed sandy soils in Egypt. Scientific J. of King Faisal Univ. (Basic and Appl. Sci.), 6: 95-110.

Marinkovic B., Starevi L., Cmobarae J., Jacimovic G. and Rajic M. (2004). By-products of sugar beet - quality animal feed. Glasnik Zastite Bilja., 27 (5): 114-118.

Mohamed H.Y. (2008). Influence of some biochemical fertilization regimes and organic fertilizer on yield and quality of some sugar beet varieties. Ph. D. Thesis, Fac. Agric., Zagazig Univ., Egypt.

Moustafa S. N., El-Sayed S. S. M., Gomaa A. M. E. and Moustafa Z. R. (2005). Response of sugar beet varieties to nitrogen and sulphur as foliar application. Egypt .J. Appl. Sci., 20 (9): 45-58.

Omar M. I. (2007). Effect of potassium and organic fertilizer on the productivity of some sugar beet varieties under saline condition. Ph.D. Thesis, Fac. Agric., Alexandria Univ., Egypt.

Ouda S. M. M. (2009). Yield and quality of two sugar beet varieties as influenced by nitrogen fertigation regimes under drip-irrigation system. J. Agric. Sci. Mansoura Univ., 34 (4): 3189-3198.

Page A.L. (1982)." Methods of Soil Analysis" Chemical and microbiological properties $\left(2{ }^{\text {nd }}\right.$ ed.). Agron. 9, Am. Soc. Agron. Inc. Publ. Madison, Wis, U.S.A.

Salama A.A. and Badawi M.A. (1996). Evaluation of six sugar beet cultivars under nitrogen levels and harvesting dates. J. Agric. Sci., Mansoura Univ., 21 (1):139-153.

Seok-In Yun and Hee-Myong Ro (2009). Natural $15 \mathrm{~N}$ abundance of plant and soil inorganic-N as evidence for over-fertilization with compost. Soil Biology \& Biochemistry, 41: 1541-1547.

Zalat S.S. and Nemeat Alla E.A.E. (2001). Yield and quality of sugar beet as affected by inorganic and organic fertilizer. Minufiya $\mathrm{J}$. Agric. Res., 26 (5):1187-1193. 


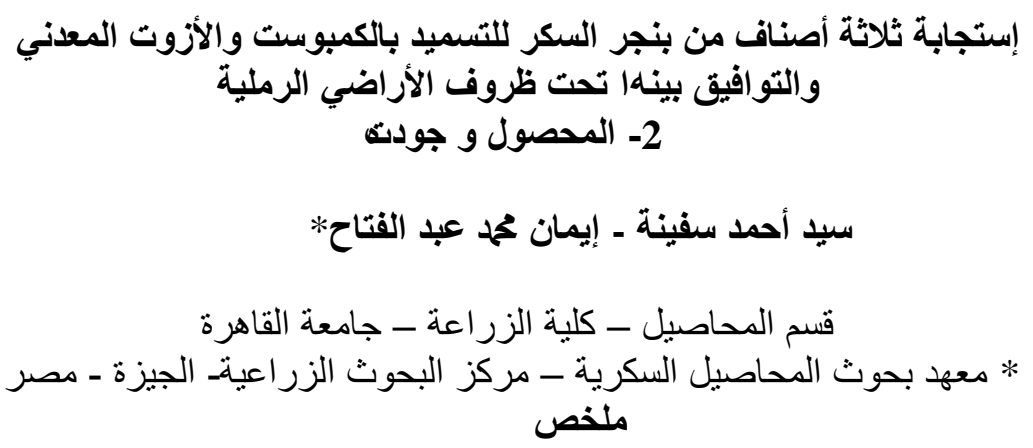

أجريت تجربتان حقليتان بمحطة التجارب الزر اعية الصحر اوية لكلية الزراعة جامعة القاهرة بو ادي النطرون،

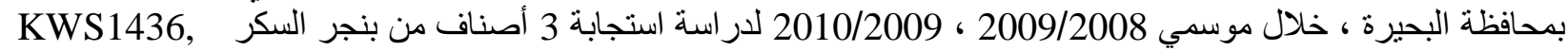
(Swello, Faraha)

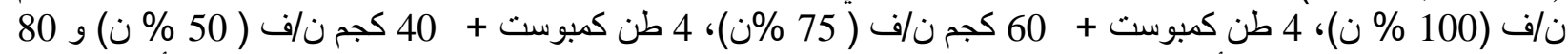

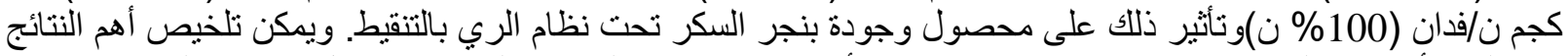

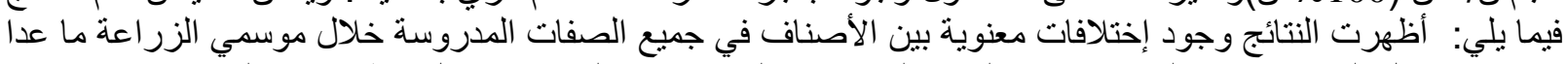

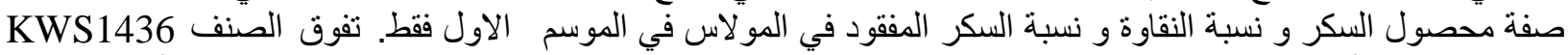

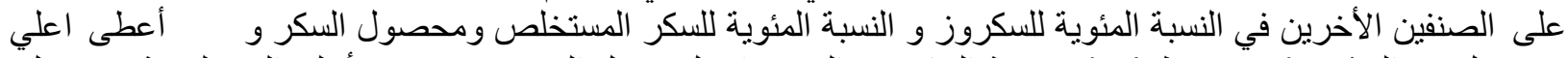

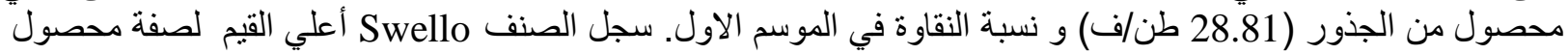

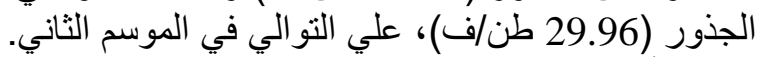

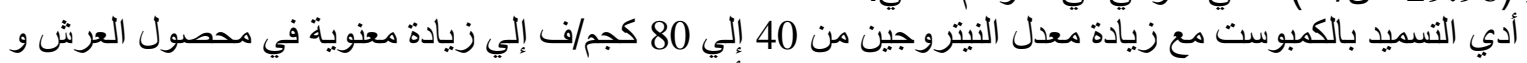

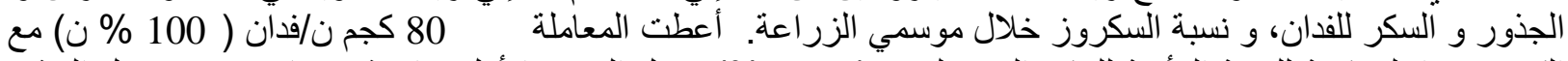

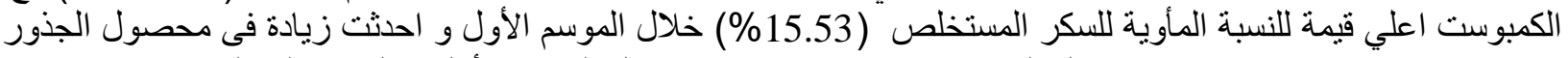

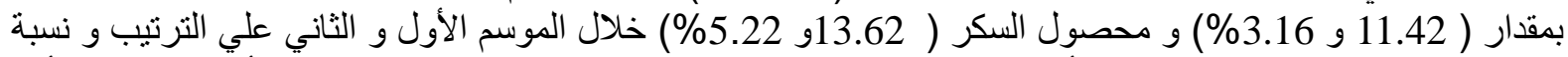

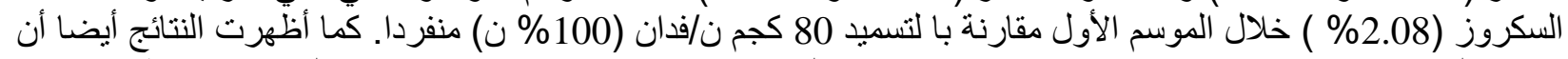

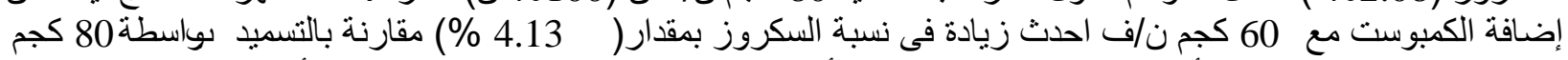

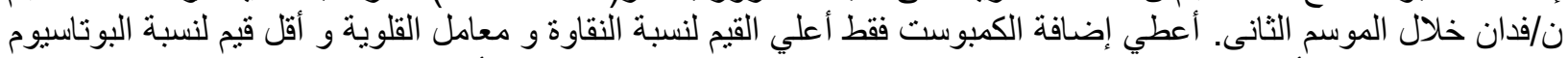

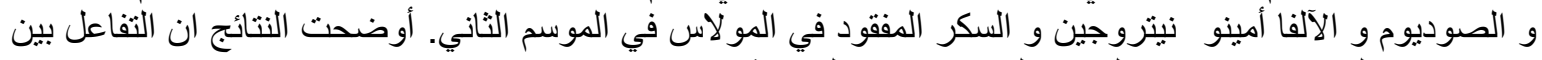

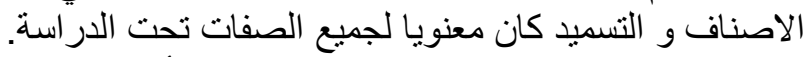

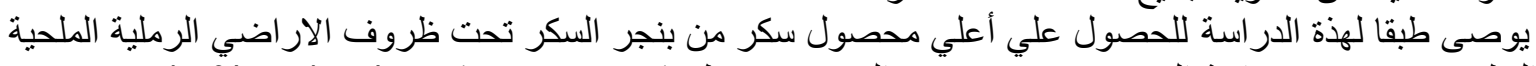

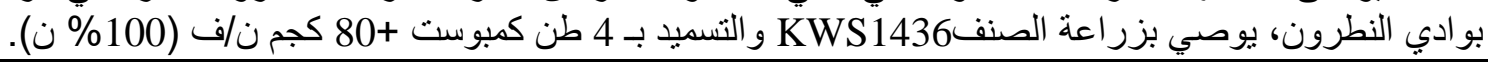
المجلة العلمية لكلية الزراعة - جامعة القاهرة - المجلد (62) العدد الرابع (أكتوبر 2011): 447-456. 